\title{
ISOLATION AND IDENTIFICATION OF CHROMOBACTERIUM SP. FROM DIFFERENT ECOSYSTEMS
}

\author{
VISHNU TS, PALANISWAMY M* \\ Department of Microbiology, Karpagam University, Coimbatore, Tamil Nadu, India. Email: m.palaniswamy@gmail.com
}

Received: 23 August 2016, Revised and Accepted: 09 September 2016

\begin{abstract}
Objectives: The study was aimed at isolating and identifying violacein-producing bacteria from the different ecosystems.

Methods: The violet color pigment (violacein) producing bacteria was isolated and identified morphologically and genomically.

Results: A total of ten bacterial Chromobacterium sp. were isolated from different sources such as soil and water. The violacein productivity was screened by the total isolates and the selected two strains were identified as Chromobacterium violaceum CV4 and Chromobacterium vaccinii CV5 by
\end{abstract} morphologically and genomically.

Conclusion: In the present study, the two isolate was morphologically and genomically identified as Chromobacterium violaceum CV4 and Chromobacterium vaccinii CV5, last one was found to be the novel strain from natural sources which possess a high violacein producing capability.

Keywords: Chromobacterium sp., Violacein, Pigment production.

(C) 2016 The Authors. Published by Innovare Academic Sciences Pvt Ltd. This is an open access article under the CC BY license (http://creativecommons. org/licenses/by/4. 0/) DOI: http://dx.doi.org/10.22159/ajpcr.2016.v9s3.14847

\section{INTRODUCTION}

Natural products have an abundant source of compounds which have proved as a useful antiviral chemotherapy for the infectious human diseases $[1,2]$ especially those originating from plant extracts and fermentation broths from soil bacteria, which provide compounds that are used directly as drugs or lead compounds for the synthesis of new medicines [3].

The purple-colored pigment, violacein [3- (1, 2-dihydro-5-(5-hydroxy1H-indol-3-yl)-2-oxo-3H-pyrrol- 3-ilydene)-1, 3-dihydro-2H-indol-2one], is produced mainly by the bacteria of genus Chromobacterium. It has attracted increased interest owing to its important biological activities and pharmacological potential. Violacein has strong antibacterial effects making it a promising candidate as an antibiotic. When administered in combination with other antibiotics, the impact is more effective against the bacteria than the use of antibiotics [4]. The pigment shows a strong anti-bacterial activity against several potentially pathogenic bacteria such as Staphylococcus, Streptococcus, Bacillus, Mycobacterium, Neisseria, and Pseudomonas. In addition, violacein has a strong bactericidal [5], antitumor [6], antiviral [7], antioxidant [8], and antiprotozoan activities [9-12]. Violacein is used as a biological dye, though it exhibits various properties [13]. Therefore, it is very much importance to discover the new microorganisms effectively producing violacein with the better biological process.

Violacein-producing bacteria encompass various genera and found in all types of natural environments, from marine to freshwater and soil environments. A possible commonality is that violacein producers are generally sessile bacteria which makes them more vulnerable to predation [14]. It leads to the idea that violacein serves as some sort of defense mechanism for producingstrain or it provides these sessile bacteria for some competitive advantage. Violacein has several kinds of bioactivity; therefore, it is very much important to discover new microorganisms effectively producing violacein with the better biological process [15].

Chromobacterium violaceum is the most studied bacterium in the violacein production field. The pigment is also produced by other bacteria, such as the psychotropic bacterium RT102 [13], Janthinobacterium lividum (formerly known as Chromobacterium lividum) [16-19] and Alteromonas luteoviolacea [20], but the yield and conditions of production are different. The main aim of the study was to isolate novel violaceinproducing bacteria from natural sources like soil and water.

\section{METHODS}

\section{Sample collection}

Thirty-three samples of agricultural soil and water samples were collected from the different localities at Trivandrum and Nedumangad (Kerala) India. Thirteen samples were taken from the agriculture soil irrigated with canal water, ten from agriculture soil irrigated with pond water, and ten water sample from the well near the paddy fields.

\section{Isolation of bacteria}

The bacteria was originally isolated by plating the dilutions of soils and water samples in the saline solution $(0.9 \% \mathrm{NaCl})$ in the nutrient agar and incubated at $37^{\circ} \mathrm{C}$ for $48 \mathrm{hrs}$. Individual colonies of bacteria which varied in shape with the violet color colonies were picked up, and the pure culture was sought by streaking in the nutrient agar. The bacterial isolates were stored in the nutrient agar at $4^{\circ} \mathrm{C}$ and subcultured every 4 weeks.

\section{Pigment production and extraction}

The production profile of crude violacein was studied by growing the strains in a $500 \mathrm{~mL}$ flask containing $200 \mathrm{~mL}$ of nutrient broth. Fermentations were carried out at $37^{\circ} \mathrm{C}$ for $48 \mathrm{hrs}$ with an inoculum size of 3\% (v/v) 24 hrs old culture (OD 660 approximately 1). After 48 hrs of the incubation period, broth was taken for the pigment extraction.

For fast and simple assay of violacein, the crude violacein separated from the cells were measured as follows: (i) aliquots of $5 \mathrm{~mL}$ fermented broth were collected, centrifuged at 10,000× $\mathrm{g}$ for 5 minutes and the supernatant was discarded, (ii) the cell pellets were then rinsed with the deionized water, followed by centrifugation $(10,000 \times \mathrm{g}$ for 5 minutes) and decanting of supernatant, (iii) $5 \mathrm{~mL}$ ethanol (with a purity of $99.7 \%$ ) was added to the pellets and the cells were disrupted by ultrasonication (200 Wand 10 minutes). The ethanol extract was separated from the cells by centrifugation at $10,000 \times \mathrm{g}$ for 5 minutes, (iv) this extraction procedure was repeated until the cells were completely bleached, and (v) all the supernatants were collected as a crude violacein for measuring the violacein concentration. The 
absorbance of the ethanol solution from the crude violacein sample was measured using an ultraviolet-visible spectrophotometer (Beckman DU800, USA) (extinction coefficient of violacein $=10.955 \mathrm{~L} /(\mathrm{g} \mathrm{cm})$ in ethanol at $570 \mathrm{~nm}$ ) [20]

\section{Identification of bacterium}

The bacterial isolates were identified on the basis of classification schemes published in Bergey's manual of systematic bacteriology [21] To identify and characterize phylogenetically, a small violet punctiform bacterial colony (designated as CV4 and CV5) was selected and identified by using $16 \mathrm{~S}$ rRNA gene sequencing analysis and a phylogenetic tree was created by the MEGASOFT program [22].

\section{RESULTS AND DISCUSSION}

Isolation and screening of the violet pigment producing bacteria Pigments are widely used in food, cloth, painting, cosmetics, pharmaceuticals, and plastics. The uncontrolled application of the synthetic pigment leads to serious health hazards and environmental pollution. Hence, it is very important to find an alternative natural pigment with the biodegradable and ecofriendly properties. Bacterial pigments possess enormous efficiency as medicinally important products Violacein, a violet pigment produced by $C$. violaceum, has attracted much attention in the recent years due to its pharmacological properties.

In this work, ten violet pigment producing bacterial species were isolated from the thirteen different samples from the various places in Trivandrum and Nedumangad, Kerala (Table 1). The isolates were numbered as CV1-CV10. The colonies were round, convex, smooth, and violet. The intensity of the purple color is increasing on prolonged incubation from light to dark purple color. The pigments were produced in colonies but not secreted when cells were grown on agar media. Soby et al. [23] discussed that the colonies were initially cream-colored, turning deep purple medial to a distal in the colony, the whole colony becoming deeply pigmented by the third day. Some isolates produce light purple color colonies. These ten isolates were examined for, the violacein production in liquid media. The results showed that the purple color pigment production was varied with the strains.

The physiological and biochemical characterization of bacterial isolates has been done to confirm the genus. Based on the morphological and partial biochemical characteristics, isolates found to be a motile, nonspore forming, Gram-negative, rod-shaped bacterium with the violet colonies in Nutrient agar, MacConkey agar, blood agar, and chocolate agar plates, which gave a catalase positive reaction as described in Table 2.

All the isolates study showed similar biochemical characterization. However, in citrate reduction and sugar fermentation studies some variations we found. Our results were similar with the study of Dall'Agnol et al. [24]. Their study stated that larger phenotypic variation was observed in the citrate test and the oxidation/fermentation of sucrose. This reflects the diversity of metabolic pathways to obtain the nutrients from the environment.

The ten isolates were screened for the violacein activity, two isolates Chromobacterium sp. CV5 and Chromobacterium sp. CV4

Table 1: Pigment producing bacteria isolated from the different sources

\begin{tabular}{ll}
\hline Isolates & Sources \\
\hline CV1 & Soil irrigated with canal water-Trivandrum \\
CV2 & Soil irrigated with canal water-Trivandrum \\
CV3 & Soil irrigated with pond water-Nedumangadu \\
CV4 & Well water near paddy field-Trivandrum \\
CV5 & Well water near paddy field-Nedumangadu \\
CV6 & Soil irrigated with pond water-Nedumangadu \\
CV7 & Soil irrigated with pond water-Nedumangadu \\
CV8 & Soil irrigated with pond water-Nedumangadu \\
CV9 & Soil irrigated with pond water-Trivandrum \\
CV10 & Well water near paddy field-Trivandrum \\
\hline
\end{tabular}

Table 2: Biochemical characterization of isolates

\begin{tabular}{|c|c|c|c|c|c|c|c|c|c|c|}
\hline Characteristics & CV1 & CV2 & CV3 & CV4 & CV5 & CV6 & CV7 & CV8 & CV9 & CV10 \\
\hline Catalse & + & + & + & + & + & + & + & + & + & + \\
\hline Oxidase & + & + & + & + & + & + & + & + & + & + \\
\hline Indole production & - & - & - & - & - & - & - & - & - & - \\
\hline Methyl red & - & - & - & - & - & - & - & - & - & - \\
\hline Voges proskeur & - & - & - & - & - & - & - & - & - & - \\
\hline Citrate & + & + & + & + & + & + & + & + & + & + \\
\hline Urease & - & - & - & - & - & - & - & - & - & - \\
\hline TSI & $\mathrm{A} / \mathrm{A}$ & $\mathrm{K} / \mathrm{A}$ & $\mathrm{A} / \mathrm{A}$ & $\mathrm{A} / \mathrm{A}$ & $\mathrm{A} / \mathrm{A}$ & $\mathrm{K} / \mathrm{A}$ & $\mathrm{K} / \mathrm{A}$ & $\mathrm{A} / \mathrm{A}$ & $\mathrm{A} / \mathrm{A}$ & $\mathrm{A} / \mathrm{A}$ \\
\hline $\mathrm{H}_{2} \mathrm{~S}$ production & - & - & - & - & - & - & - & - & - & - \\
\hline Lysine decarboxylase & - & - & - & - & - & - & - & - & - & - \\
\hline Arginine dihydrolase & + & + & + & + & + & + & + & + & + & + \\
\hline Ornithine decarboxylase & - & - & - & - & - & - & - & - & - & - \\
\hline Aesculin hydrolysis & - & - & - & - & - & - & - & - & - & - \\
\hline \multicolumn{11}{|l|}{ Fermentation/oxidation } \\
\hline Glucose & + & + & + & + & + & - & + & - & - & + \\
\hline Isositol & - & - & - & - & - & - & - & - & - & - \\
\hline Sorbitol & - & - & - & - & - & - & - & - & - & - \\
\hline Rhamnose & - & - & - & - & - & - & - & - & - & - \\
\hline Sucrose & - & - & - & - & - & - & - & + & + & - \\
\hline Melobiose & - & - & - & - & - & - & - & - & - & - \\
\hline Arabinose & - & - & - & - & - & - & - & - & - & - \\
\hline
\end{tabular}




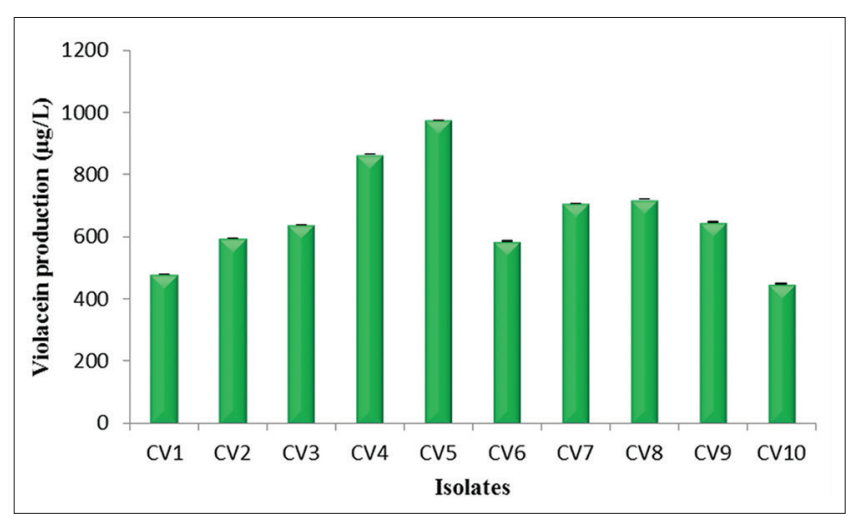

Fig. 1: Violacein production by the ten different isolates

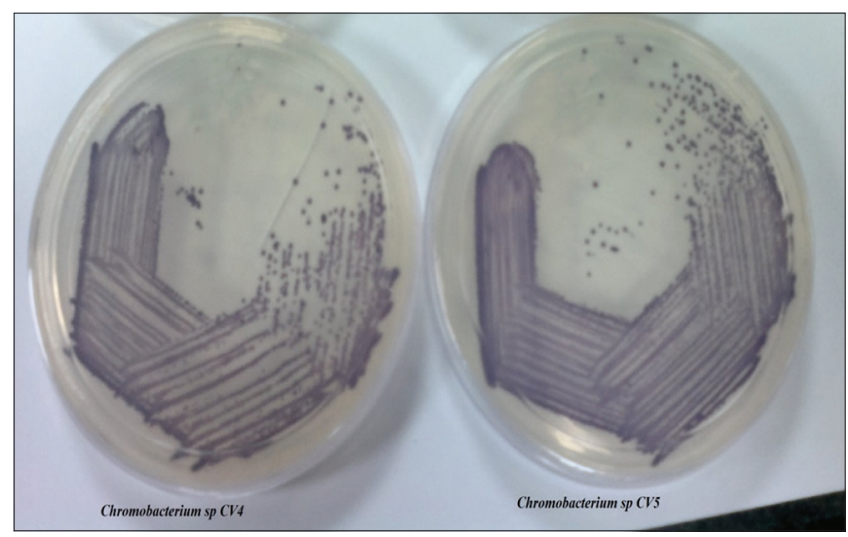

Fig. 2: Chromobacterium sp. CV4, Chromobacterium sp. CV5 showed the high violacein activity of $975 \mu \mathrm{g} / \mathrm{L}$ and $865 \mu \mathrm{g} / \mathrm{L}$. Very low violacein activity was found in the case of Chromobacterium sp. CV10 (449 $\mu \mathrm{g} / \mathrm{L})$ and CV1 (479 $\mu \mathrm{g} / \mathrm{L})$ (Fig. 1). The two isolates Chromobacterium sp. CV4, Chromobacterium sp. CV5 (Fig. 2) were used for the further study.

\section{Identification of violacein producing isolates}

The two isolates, the 16S rDNA gene sequences of strains (Fig. 3) (CV4 and CV5) were compared with the sequence to determine the relatedness of strains at the genetic level and the entire 16S rDNA gene was sequenced from the each isolate. The sequence of $1244 \mathrm{bp} 16 \mathrm{~S} \mathrm{rDNA}$ of the isolated bacteria showed $100 \%$ similarity to C. violaceum for the isolate Chromobacterium CV4 and 99\% similarity to Chromobacterium vaccinii for Chromobacterium CV5.

Molecular method was used for obtaining more taxonomic information. DNA was isolated from the culture (isolate CV4 and CV5). Quality was evaluated on $1.2 \%$ agarose gel; a single band of high-molecular weight DNA has been observed (Fig. 4).

Phylogenetic tree (Figs. 5 and 6) constructed by MEGA SOFT showed that the isolated bacterial culture CV5 is closely related to the $C$. vaccinii strain KJ806485 and CV4 is related to C. violaceum strain KJ806351. This study mainly revealed the isolation of $C$. violaceum $\mathrm{CV} 4$ and new strain C. vaccinii CV5 in the Indian ecosystems. Previously this strain, C. vaccinii was discussed in the Soby et al. [23], they isolated it from the irrigation ponds associated with the wild and cultivated cranberry bogs in Massachusetts, USA. Here, we also isolated this novel strain similar to those ecosystems (well water near paddy field from Nedumangad). The key point is that the violacein-violet pigment produced by the various species is yet to be exploited and great potential in the various fields of cancer biology, biotechnological, industrial and clinical microbiology [23]. The selected two isolates would be used for

>SR2 64-CV4-16S

GGTGAGTAATGCGTCGGAATGTACCGTGTAATGGGGGATAGCTCGGCGAAAGCCGGATTAATACCGCATACGCCCTG AGGGGGAAAGCGGGGGATCGAAAGACCTCGCGTTATACGAGCAGCCGACGTCTGATTAGCTAGTTGGTGAGGTAAGA GCTCACCAAGGCGACGATCAGTAGCGGGTCT GAGAGGATGATCCGCCACACTGGGACTGAGACACGGCCCAGACTCC TACGGGAGGCAGCAGTGGGGAATTTTGGACAATGGGGGCAACCCTGATCCAGCCATGCCGCGTGTCTGAAGAAGGCC TTCGGGTTGTAAAG GACTTTTGTCAGGGAGGAAATCCCGCTGGT TAATACCCGGCGGGGATGACAGTACCTGAAGAA TAAGCACCGGCTAACTACGTGCCAGCAGCCGCGGTAATACGTAGGGTGCGAGCGTTAATCGGAATTACTGGGCGTAA AGCGTGCGCAGGCGGTTGTGCAAGTCTGATGTGAAAGCCCCGGGCTTAACCTGGGAACGGCATTGGAGACTGCACAG CTAGAGT GCGTCAGAGGGGGGTAGAATTCACGTGTAGCAGTGAAATGCGTAGAGATGTGGAGGAATACCGATGGCG AAGGCAGCCCCCTGGGATGACACTGACGCTCATGCACGAAAGCGTGGGGAGCAAACAGGATTAGATACCCTGGTAGT CCACGCCCTAAACGATGTCAACTAGCTGTTGGGGGTTTGAATCCTTGGTAGCGTAGCTAACGCGTGAAGTTGACCGC CTGGGGAGTACGGCCGCAAGGTTAAAACTCAAAGGAATTGACGGGGACCCGCACAAGCGGTGGATGATGTGGATTAA TTCGATGCAACGCGAAAAACCTTACCTGCTCTTGACATGTACGGAACTTGCCAGAGATGGCTTGGTGCCCGAAAGGG AGCCGTAACACA GGTGTGCATGG CTGTCGTCAGCTCGT GTCGTGAGATGT TGGGT TAAGTCCCGCAACGAGCGCAA CCСT TGT CATTAGT TGCCATCATTAAGTTGGGCACTCTAAT GAGACTGCCGGTGACAAA CCGGAGGAAGGTGGGGAT GACGTCAAGTCCTCATGGCCCTTATGAGCAGGGCTTCACACGTCATACAATGGTCGGTACAGAGGGTTGCCAAGCCG CGAGGTGGAGCTAATCTCAGAAAACCGATCGTAGTCCGGATCGCACTCTGCAACTCGAGTGCGTGAAGTCGGAATCG CTAGTAATCGCA

>SR264-CV5-16S

GGTGAGTAATGCGTCGGAATGTACCGTGTAATGGGGGATAGCTCGGCGAAAGCCGGATTAATACCGCATACGCCCTG AGGGGGAAAGTGGGGGACCGTAAGGCCTCACGTTATACGAGCAGCCGACGTCTGATTAGCTAGTTGGTGAGGTAAAG GCTCACCAAGGCGACGATCAGTAGCGGGTCT GAGAGGATGATCCGCCACACTGGGACTGAGACACGGCCCAGACTCC TACGGGAGGCAGCAGTGGGGAATTTTGGACAATGGGCGCAAGCCTGATCCAGCCATGCCGCGTGTCTGAAGAAGGCC TTCGGGTTGTAAAGGACTTTTGTTCGGGAGGAAATCCCGCTGGTTAATACCTGGCGGGGATGACAGTACCGGAAGAA TAAGCACCGGCTAACTACGTGCCAGCAGCCGCGGTAATACGTAGGGTGCAAGCGTTAATCGGAATTACTGGGCGTAA AGCGTGCGCAGGCGGTTGTGCAAGTCTGATGTGAAAGCCCCGGGCT TAACCTGGGAACGGCATTGGAGACTGCACGA CTAGAGTGCGTCAGAGGGGGGTAGAATTCCACGT GTAGCAGTGAAATGCGTAGAGATGTGGAGGAATACCGATGGCG AAGGCAGCCCCCTGGGATGACACTGACGCTCATGCACGAAAGCGTGGGGAGCAAACAGGATTAGATACCCTGGTAGT CCACGCCCTAAACGATGTCAACTAGCTGTTGGGGGTTTGAATCCTTGGTAGCGTAGCTAACGCGTGAAGTTGACCGC CTGGGGAGTACGGCCGCAAGGTTAAAACT CAAAGGAATTGACGGGGACCCGCACAAGCGGTGGATGATGTGGATTAA TTCGATGCAACGCGAAAAACCTTACCTGCTCTTGACATGTACGGAACTTGCCAGAGATGGCTTGGTGCCCGAAAGGG AGCCGTAACACAGGTGCTGCATGGCTGTCGTCAGCTCGT GT CGTGAGAT GT TGGGTTAAGTCCCGCAACGAGCGCAA CCCTTGTCATTAGTTGCCATCATTCAGTTGGGCACTCTAAT GAGACTGCCGGTGACAAACCGGAGGAAGGTGGGGAT GACGTCAAGTCCTCATGGCCCTTATGAGCAGGGCTTCACACGTCATACAATGGTCGGTACAGAGGGTTGCCAAGCCG CGAGGTGGAGCTAATCT CAGAAAACCGATCGTAGTCCGGAT CGCACTCTGCAACTCGAGTGCGTGAAGT CGGAATCG CTAGTAATCGCA

Fig. 3: 16SrDNA sequence of Chromobacterium CV4 and CV5 


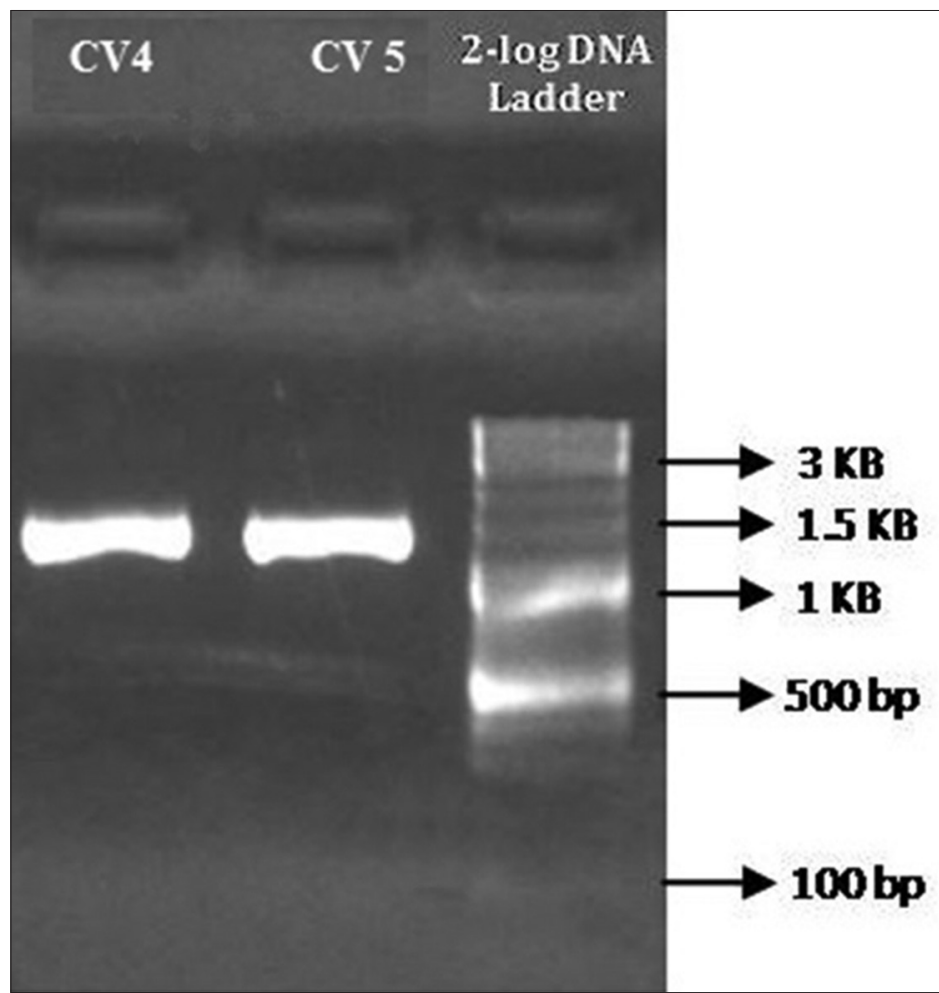

Fig. 4: Polymerase chain reaction amplification of 16SrDNA amplicon on agarose gel

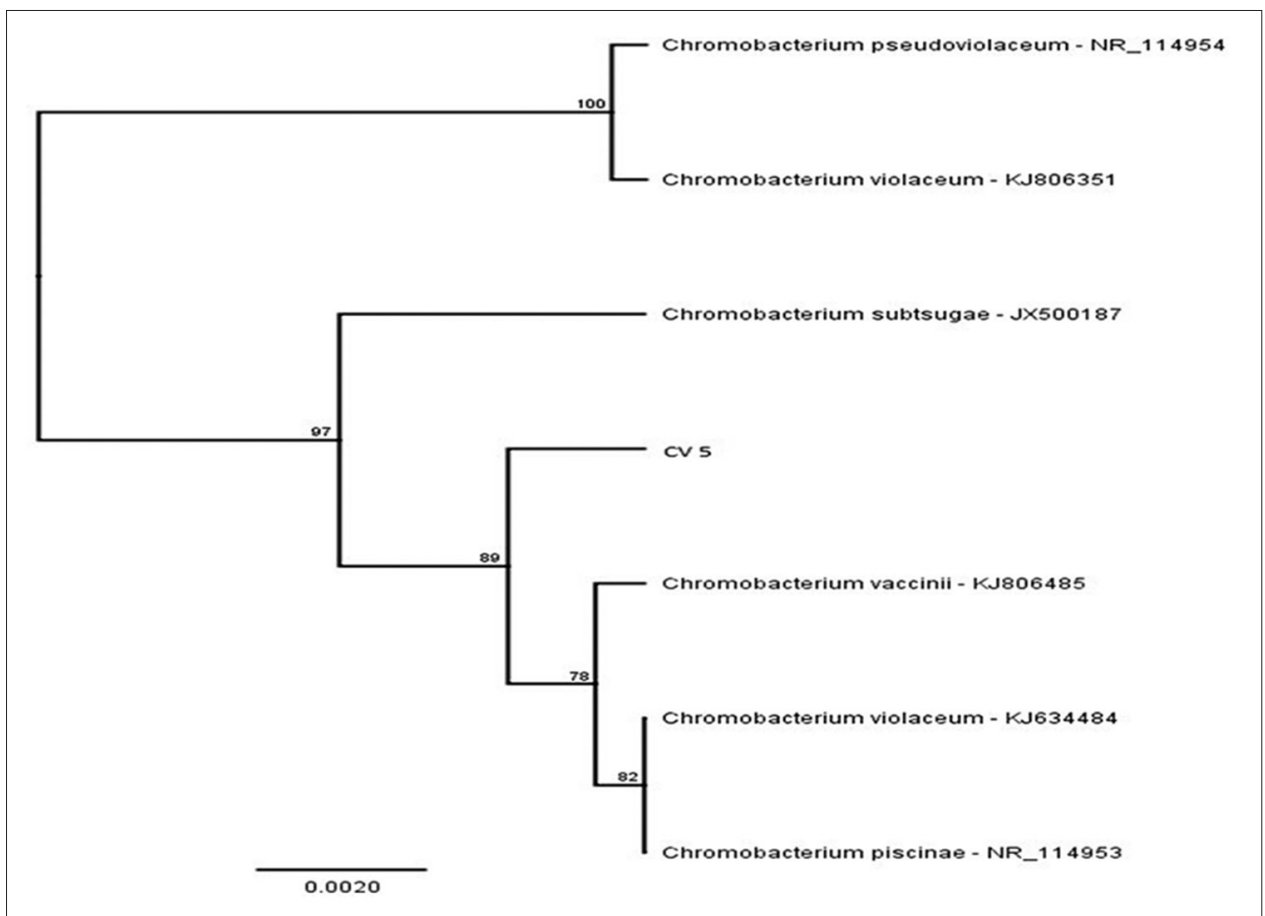

Fig. 5: Phylogenetic tree for the isolate, Chromobacterium CV4

further studies on violacein including its therapeutical and industrial application.

\section{CONCLUSION}

Biopigments produced by bacteria possess enormous efficiency in medicinally important products. Violacein, the major pigment produced by $C$. violaceum, has been shown to have antibiotic, antitumoral, and anti-
Trypanosoma activities. In the present work, an attempt was made to isolate new violacein producing bacteria from the soil and water samples. On the basis of phylogenetic tree construction, we propose strain CV5 as the type strain of a novel species of the genus Chromobacterium, (C. vaccinii CV5) which is first isolated from Kerala ecosystems. From ten isolates, two isolates $C$. vaccinii $\mathrm{CV} 5$ and $C$. violaceum $\mathrm{CV} 4$ were selected for the further study which the warrants further investigation. 


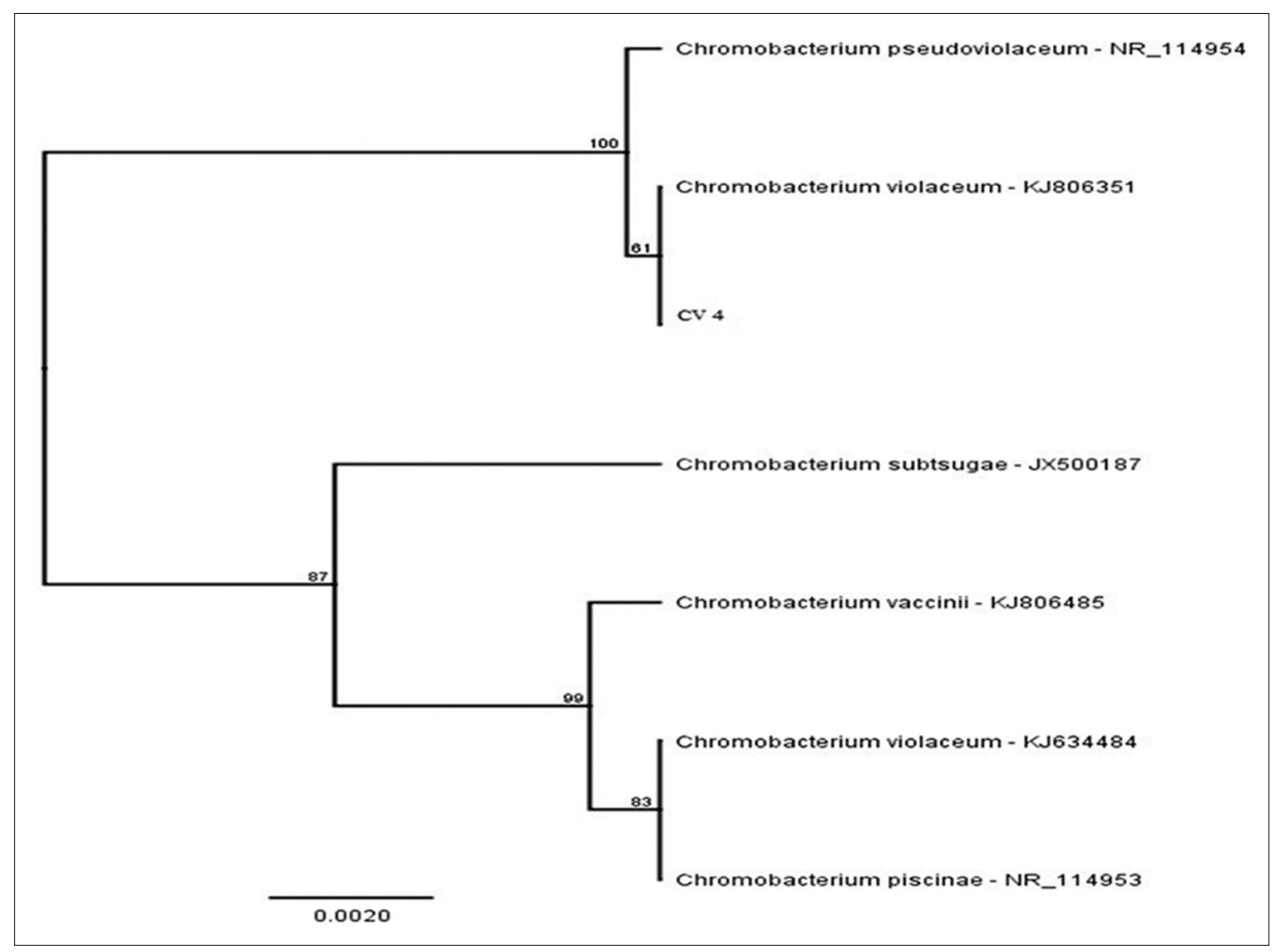

Fig. 6: Phylogenetic tree for the isolate, Chromobacterium CV5

\section{REFERENCES}

1. Pujol CA, Errea MI, Matulewicz MC, Damonte EB. Antitherapeutic activity of S1, an algal derived sulfated galactan. Phytother Res 1996;10(5):410-3.

2. Bedoya LM, Sanchez-Palomino S, Abad MJ, Bermejo P, Alcami J. Anti-HIV activity of medicinal plant extracts. J Ethnopharmacol 2001;77(1):113-6.

3. Nielsen J. Combinatorial synthesis of natural products. Curr Opin Chem Biol 2002;6(3):297-305.

4. Subramaniam S, Ravi V, Sivasubramanian A. Synergistic antimicrobia profiling of violacein with commercial antibiotics against pathogenic micro-organisms. Pharm Biol 2014;52(1):86-90.

5. Duran N, Erazo S, Campos V. Bacterial chemistry-II: Antimicrobial photoproduct from pigment of Chromobacterium violaceum. An Acad Bras Cienc 1983;55(3):231-4.

6. Duran N, Melo PS, Haun M. In vitro Evalution of Violacein on AIDSrelated Lymphoma and Human Tumor Cell Lines, In: Proceedings of the XXV Annual Meetings of the Brazilian Society of Biochemistry and Molecular Biology, Sociedade Brasileira de Bioquímica e Biologia Molecular SBBq, Caxambu, MG, Brazil; 1996. p. 150.

7. Rettori D, Duran N. Production, extraction and purification of violacein: An antibiotic pigment produced by Chromobacterium violaceum. World J Microbiol Biotechnol 1998;14:685-8.

8. De Azevedo MB, Alderete J, Rodriguez JA, Souza AO, Rettori D, Torsoni MA, et al. Biological activities of violacein, a new antitumoralindole derivative, in an inclusion complex with - cyclodextrin. J Incl Phenom Macrocycl Chem 2000;37(1-3):93-101.

9. Durán N, Antonio RV, Haun M, Pilli RA. Biosynthesis of a trypanocide by Chromobacterium violaceum. World J Microbiol Biotechnol 1994;10(6):686-90.

10. Bromberg N, Duran N. Violacein transformation by peroxidases and oxidases: Implications on its biological properties. Mol Catal B Enzym 2001;11:463-7.

11. Leon LL, Miranda CC, De Souza AO, Durán N. Antileishmanial activity of the violacein extracted from Chromobacterium violaceum. J Antimicrob Chemother 2001;48(3):449-50.

12. Matz C, Deines P, Boenigk J, Arndt H, Eberl L, Kjelleberg S, et al. Impact of violacein-producing bacteria on survival and feeding of bacterivorous nanoflagellates. Appl Environ Microbiol 2004;70(3):1593-9.

13. Shirata A, Tsukamoto T, Yasui H, Hata T, Hayasaka S, Kojima A et al. Isolation of bacteria producing bluish-purple pigment and use for dyeing. Jpn Agric Res Q 2000;34(2):131-40.

14. Matz C, Webb JS, Schupp PJ, Phang SY, Penesyan A, Egan S, et al. Marine biofilm bacteria evade eukaryotic predation by targeted chemical defense. PLoS One 2008;3(7):e2744.

15. Choi ST, Yoon KY, Lee JI, Robert JM. Violacein: Properties and production of a versatile bacterial pigment. BioMed Res Int 2015;2015:Article ID: 465056, 1-8.

16. Nakamura Y, Asada C, Sawada T. Production of antibacterial violet pigment by psychrotrophic bacterium RT102 strain. Biotechnol Bioprocess Eng 2003;8:37-40.

17. Shirata A, Tsukamoto T, Yasui HK, Hayasaka S, Kojima A. Production of bluish-purple pigments by Janthinobacterium lividum isolated from the raw silk and dyeing with them. J Sericult Sci Jpn 1997;66(6):377-85.

18. Tobie WC. The pigment of Bacillus violaceus: I. The production, extraction, and purification of violacein. J Bacteriol 1935;29(3):223-7.

19. Laatsch H, Thomson RH, Philip JC. Spectroscopic properties of violacein and related compounds: Crystal structure of tetramethylviolacein. J Chem Soc Perkin Trans 1984;2:1331-9.

20. Wang HS, Jiang PX, Lu Y, Ruan Z, Jiang R, Xing XX, et al. Optimization of culture conditions for violacein production by a new strain of Duganella sp. B2. Biochem Eng J 2009;44(2-3):119-24.

21. Krieg NR, Holt JG. Bergey's Manual of Systematic Bacteriology. Baltimore and London: Williams \& Wilkins; 1984-1989.

22. Wilson K. Preparation of genomic DNA from bacteria. In: Ausubel FM, Brent R, Kingston RE, Moore DD, Seidman JG, Smith JA, et al., editors. Current Protocols in Molecular Biology. New York: John Wiley \& Sons; 1987. p. 2.4.1-5.

23. Soby SD, Sudhindra RG, Contreras C, Frank LC. Chromobacterium vaccinii sp. Nov., Isolated from native and cultivated cranberry (Vaccinium macrocarpon Ait.) Bogs and irrigation ponds. Int J Syst Evol Microbiol 2013;63:1840-6.

24. Dall'Agnol LT, Martins RN, Vallinoto AC, Ribeiro KT. Diversity of Chromobacterium violaceum isolates from aquatic environments of state of Pará, Brazilian Amazon. Mem Inst Oswaldo Cruz 2008;103(7):678-82. 